\title{
Diabetes em foco: relato de experiência sobre projeto de extensão universitária durante a pandemia da COVID-19
}

\author{
Diabetes in focus: experience report on university extension project during the COVID-19 \\ pandemic
}

Diabetes en foco: informe de experiencia en proyecto de extensión universitaria durante la pandemia COVID-19

\begin{abstract}
Beatrice Sousa Alencar ${ }^{1 *}$, Ana Carolina Mourão Barreto ${ }^{1}$, Indira Luz da Silva ${ }^{1}$, João Victor Coimbra França $^{1}$, Rafael dos Santos Nunes ${ }^{1}$, Suãyne Regina Reitz ${ }^{1}$, Susana Aparecida Silveira ${ }^{1}$, Felipe Scipião Moura², Lucielma Salmito Soares Pinto ${ }^{1}$, Luciana Tolstenko Nogueira'.
\end{abstract}

\section{RESUMO}

Objetivo: Detalhar as atividades remotas de educação em saúde para o público diabético, durante a pandemia da COVID-19, organizadas por discentes e docentes do curso de medicina de uma universidade pública no estado do Piauí. Relato de experiência: De janeiro a agosto de 2020, aconteceram capacitações para os discentes lidarem adequadamente com o público alvo. Assim, até dezembro de 2020, foi abordado público alvo de 30 participantes, dos quais 14 tinham Diabetes Mellitus Tipo II, 10 tinham Diabetes Mellitus Tipo I e 6 eram familiares de diabéticos. Vídeos informativos foram criados. Reuniões virtuais com profissionais da saúde ocorreram. 20 participantes tiveram suas demandas individuais melhor gerenciadas pelo sistema de apadrinhamento. Dentre eles, 8 estavam com dificuldades dietéticas, $7 \mathrm{com}$ problemas nas atividades físicas, 5 sem um acompanhamento periódico endocrinológico e 1 apresentava alterações psicológicas, pelo medo das consequências da doença. Considerações Finais: Os participantes enfatizaram a importância do sistema de apadrinhamento e relataram que adotaram hábitos mais saudáveis, mas que tiveram dificuldades devido ao contexto da pandemia. Os alunos desenvolveram habilidades para a relação médico-paciente, sobretudo pela aplicação de técnicas de abordagem comportamental, bem como pelo desenvolvimento de uma escuta empática e humanizada da realidade de cada indivíduo.

Palavras-chave: Diabetes mellitus, Educação em saúde, COVID-19.

\begin{abstract}
Objective: Detailing the remote activities of health education for the diabetic public, during the COVID-19 pandemic, organized by students and professors of the medical course of the public university in the state of Piauí. Experience report: From January to August 2020, training was made for students to properly deal with the target audience. Thus, until December 2020, a target audience of 30 participants was approached, of which 14 had Type II Diabetes Mellitus, 10 had Type I Diabetes Mellitus and 6 were relatives of diabetics. Informative videos have been created. Virtual meetings with health professionals took place. 20 participants had their individual demands better managed by the sponsoring system. Among them, 8 had dietary difficulties, 7 with problems in physical activities, 5 without periodic endocrine follow-up and 1 presented psychological alterations, from fear of the consequences of the disease. Final considerations: The participants emphasized the importance of the sponsoring system and reported the adoption of healthier habits, however, they mentioned difficulties related to the context of the pandemic. The students developed skills towards the doctorpatient relationship, especially by applying behavioral approach techniques, as well as by the development of an empathic and humanized listening of the reality of each individual.
\end{abstract}

Keywords: Diabetes mellitus, Health education, COVID-19.

1 Universidade Estadual do Piauí (UESPI), Teresina - PI. *E-mail: beatricesousalencar083@gmail.com

2 Centro Universitário UNINOVAFAPI, Teresina - PI. 


\section{RESUMEN}

Objetivo: Detallar las actividades remotas de educación sanitaria para el público diabético durante la pandemia COVID-19, organizada por estudiantes y profesores del curso médico universidad pública en el estado de Piauí. Informe de experiencia: De enero a agosto de 2020, se hizo una capacitación para que los estudiantes se ocuparan adecuadamente con el público objetivo. Así, hasta diciembre de 2020, se abordó un público objetivo de 30 participantes, de los cuales 14 tenían Diabetes Mellitus Tipo II, 10 Diabetes Mellitus Tipo I y 6 eran parientes de diabéticos. Se han creado vídeos informativos. Se llevaron a cabo reuniones virtuales con profesionales de la salud. 20 participantes tenían sus demandas individuales mejor gestionadas por el sistema patrocinador. Entre ellos, 8 tenían dificultades dietéticas, 7 con problemas en las actividades físicas, 5 sin seguimiento endocrino periódico y 1 tenía alteraciones psicológicas, por temor a las consecuencias de la enfermedad. Consideraciones finales: Los participantes enfatizaron la importancia del sistema de apadrinamiento e informaron que adoptaron hábitos más saludables, pero que tenían dificultades por el contexto de la pandemia. Los estudiantes desarrollaron habilidades para la relación médico-paciente, especialmente aplicando técnicas de abordaje conductual, así como desarrollando una escucha empática y humanizada de la realidad de cada individuo.

Palabras clave: Diabetes mellitus, Educación para la salud, COVID-19.

\section{INTRODUÇÃO}

Diabetes Mellitus (DM) é uma condição crônica que ocorre em indivíduos com elevados níveis de glicose no sangue, seja por falha na produção de insulina ou falha na atividade da mesma. A insulina é um hormônio secretado pelo pâncreas que auxilia no transporte da glicose para o interior da célula, a fim de que esta seja transformada em energia (SBD, 2019).

Há dois tipos principais de diabetes, a do tipo 1, decorrente de uma falha no sistema imunológico do paciente que ataca as células beta produtoras de insulina, e a do tipo 2, que deriva de um mecanismo de resistência à insulina, no qual há um desequilíbrio entre a quantidade de insulina produzida e o seu funcionamento, o que gera uma incapacidade das células do corpo de responderem corretamente à insulina. Esse último tipo tem ocorrido cada vez mais em crianças e adultos jovens devido ao aumento dos níveis de obesidade, sedentarismo e dieta inadequada (FID, 2019).

No Brasil, estima-se que 16,8 milhões de adultos sejam portadores da doença. Ademais, o país é o terceiro no ranking de crianças e adolescentes com diabetes tipo I, totalizando cerca de 96.000. É importante salientar que grande parte dos casos diagnosticados de diabetes tipo 2 poderiam ser evitados com a adoção de hábitos de vida saudáveis (FID, 2019).

Diante do exposto, sabe-se que o tratamento da doença engloba mudanças de hábitos alimentares, prática de exercícios físicos e uso de medicações. Logo, para uma boa adesão ao tratamento e uma mudança efetiva de comportamento e de estilo de vida, é necessário desenvolver habilidades, fomentar o conhecimento e a prática do autocuidado (FARIA H, et al., 2014).

No entanto, um estudo realizado na Estratégia de Saúde da Família em um município do interior de Minas Gerais demonstrou que a adesão ao tratamento ocorre de forma mais satisfatória em relação aos medicamentos e à prática de atividades físicas, enquanto a adesão ao plano alimentar se deu em uma parcela pequena do grupo estudado, isso se deve em parte à indicação, por alguns profissionais, de mudanças bruscas na alimentação, por meio de dietas restritivas. Dessa forma, a adaptação do usuário ao plano alimentar é um dos maiores desafios dentro da Atenção Primária, sendo necessário um suporte ainda maior à promoção de ações que visem fortalecer uma adaptação a uma mudança de comportamento alimentar (FARIA H, et al., 2014).

Segundo Cruz SC (2005), a má adesão ao tratamento possui inúmeras consequências a longo prazo, como a associação de comorbidades que, consequentemente, elevam os custos do tratamento, aumentam a chance de hospitalizações e reduzem a qualidade de vida e, dessa forma, demandam mais que o dobro de cuidados necessários comparado aos cuidados com pessoas não diabéticas. Destaca-se, também, que os 
indivíduos com DM tipo 2 tem como sua maior causa de mortalidade a doença cardiovascular, de forma que esse grupo apresenta de duas a quatros vezes uma maior propensão a morrer por doença cardíaca em relação aos não diabético (CORTEZ DN, et al., 2015).

Bem como, sabe-se que a dislipidemia, a hiperglicemia, a hipertensão arterial sistêmica e o tabagismo são importantes fatores envolvidos no desenvolvimento de tais complicações crônicas na DM tipo 2. Desse modo, é importante que o paciente esteja totalmente familiarizado com as informações da doença e com os meios de obter um estilo de vida mais saudável e, dessa forma, consiga projetar as mudanças necessárias para uma melhor qualidade de vida (CORTEZ DN, et al., 2015; ZAGURY L, et al.,1987).

É preciso compreender que o conhecimento do paciente diabético, acerca de sua doença é a base do cuidado para se conseguir o auto manejo do diabetes, mas a aquisição do conhecimento, necessariamente, não se traduz em mudança de comportamento. É necessário também dar ênfase ao papel da família e conhecer melhor o paciente a respeito de seus sentimentos e suas necessidades para que se possa, assim, iniciar uma orientação específica através do estabelecimento de um vínculo efetivo e com metas para alcançar o controle do DM (OTERO LM, et al., 2008; SANTOS ECB, et al., 2005).

Assim, o projeto "Diabetes Mellitus em foco" surgiu com o intuito de proporcionar um suporte ao desenvolvimento do autocuidado, através de práticas mais saudáveis de alimentação e exercícios físicos, a pacientes atendidos em uma UBS na cidade de Teresina-PI, com ênfase no acompanhamento colaborativo nos diferentes estágios de motivação e mudança de atitude frente ao enfrentamento da DM. Entretanto, devido a pandemia do coronavírus, houve a necessidade de adaptação do projeto à modalidade remota, com a adoção de mídias digitais como ferramentas importantes e fundamentais para que se pudesse ter um seguimento viável seguindo seus objetivos (BOMFIM A, 2020).

Esse relato, tem por objetivo principal descrever as atividades remotas de educação em saúde para o público diabético, realizadas durante a pandemia da COVID-19, que foram organizadas por discentes e docentes do curso de medicina de uma universidade pública no estado do Piauí, com ênfase na estruturação de um processo de educação em saúde para o público específico, o qual se caracterizou pela disseminação do conhecimento acerca da DM e na promoção de mudança do estilo de vida desses pacientes, por meio de encontros e rodas de conversa on-line.

\section{RELATO DE EXPERIÊNCIA}

Desenvolveu-se o projeto de extensão intitulado "Diabetes em Foco", que consistiu em atividades remotas de educação em saúde para o público diabético, durante o período de janeiro a dezembro de 2020 , com a participação de 30 discentes e 2 docentes do curso de medicina de uma universidade pública no estado do Piauí. O público-alvo foi composto por 30 indivíduos, dos quais 14 possuíam DM Tipo II, 10, DM Tipo I e 6 eram familiares de pacientes diabéticos. A faixa etária variou de 4 a 71 anos de idade, e a maioria residia em Teresina, embora tivéssemos participantes de outros estados, como Santa Catarina e Minas Gerais.

O projeto foi estruturado em quatro fases: capacitações dos discentes, criação de conteúdos digitais, reuniões online e apadrinhamento. As capacitações abordaram a Diabetes Mellitus e Técnicas de Abordagem Comportamental, como entrevista motivacional e grupos operativos, a fim de prepará-los para atuar com os participantes diabéticos.

$\mathrm{Na}$ fase de criação de conteúdos digitais, foram iniciadas as divulgações em redes sociais para a formação do grupo de diabéticos, para isso foram criados perfis no Facebook e Instagram destinados a compartilhar os conteúdos do projeto, as inscrições foram realizadas por meio do Google Forms. Os participantes foram incluídos em um grupo de WhatsApp, espaço destinado para informar sobre as reuniões online e compartilhar informações sobre diabetes. Nesse período também foram produzidas seis videoaulas que abordaram temáticas introdutórias sobre a doença, a fim de embasar futuras discussões realizadas nos encontros virtuais.

$\mathrm{Na}$ terceira fase, foram realizadas oito reuniões online, por meio do aplicativo Google Meet, cada encontro foi preparado por um grupo de estudantes e contou com a participação de profissionais da área da saúde, 
como dois médicos endocrinologistas, médico oftalmologista, psicólogo, fisioterapeuta, educadora física, enfermeira, cirurgião-dentista e nutricionista (Quadro 1). As temáticas foram abordadas por meio de dinâmicas, em que os participantes poderiam interagir tanto pelo chat, como pelo aplicativo Vevox.

Quadro 1 - Descrição das reuniões online.

\begin{tabular}{|c|c|}
\hline TEMA DA REUNIÃO & DESCRIÇÃO \\
\hline Mitos e Verdades da DM & $\begin{array}{l}\text { Abordagem de assuntos essenciais para } \\
\text { compreender melhor a fisiopatologia da doença, } \\
\text { como por exemplo: definição da doença, sintomas, } \\
\text { complicações crônicas, como proceder em casos } \\
\text { de hiperglicemia e hipoglicemia, dificuldades em } \\
\text { controlar a glicemia e medicamentos. }\end{array}$ \\
\hline Alimentação Saudável & $\begin{array}{l}\text { Debate sobre as dificuldades em seguir as } \\
\text { orientações sobre alimentação saudável; } \\
\text { importância das fibras na alimentação; mitos da } \\
\text { alimentação; diet e light; adoçantes; periodicidade } \\
\text { das refeições. }\end{array}$ \\
\hline Exercício Físico & $\begin{array}{l}\text { Orientações sobre temas fundamentais para a } \\
\text { prática de exercícios físicos pelos diabéticos: } \\
\text { cuidados com a glicemia antes, durante e após as } \\
\text { atividades; bem como os melhores horários para a } \\
\text { prática e os tipos de exercícios físicos, aeróbicos e } \\
\text { de resistência. }\end{array}$ \\
\hline Aspectos Emocionais & $\begin{array}{l}\text { Uma conversa sobre as dificuldades de conviver } \\
\text { com as especificidades e tratamento da Diabetes; } \\
\text { bem como a abordagem de ferramentas que } \\
\text { auxiliam na saúde mental, como a prática de } \\
\text { meditação, Reike e aromaterapia. }\end{array}$ \\
\hline Complicações da Doença & $\begin{array}{l}\text { Destinada a explicar e sanar dúvidas sobre as } \\
\text { principais complicações da Diabetes, como a } \\
\text { retinopatia, neuropatia diabética, hiperglicemia e } \\
\text { hipoglicemia; e sobretudo alertar quanto à } \\
\text { prevenção de tais agravos. }\end{array}$ \\
\hline Autocuidado dos Pés Diabéticos & $\begin{array}{l}\text { Abordagem do auto cuidado indispensável com os } \\
\text { pé diabético como o uso de sandálias apropriadas, } \\
\text { não andar descalços, ficar atento a calos e feridas. }\end{array}$ \\
\hline Análise de Situações-Problema & $\begin{array}{l}\text { Reunião focada em sanar possíveis dúvidas e } \\
\text { avaliar o conhecimento dos participantes, por meio } \\
\text { da construção de soluções na análise de situações } \\
\text { problema e explanação de novas ferramentas } \\
\text { digitais para o acompanhamento de pacientes } \\
\text { diabéticos. }\end{array}$ \\
\hline Encerramento & $\begin{array}{l}\text { Análise de impacto do projeto na vida dos } \\
\text { participantes, por meio de um questionário de } \\
\text { avaliação do projeto e um espaço de fala para } \\
\text { trocas de experiência. Além disso, foi feito uma } \\
\text { retrospectiva dos assuntos abordados, por meio de } \\
\text { dinâmicas. }\end{array}$ \\
\hline
\end{tabular}

Fonte: Alencar BS, et al., 2021.

$\mathrm{Na}$ fase apadrinhamento, que ocorreu a partir da 5을 reunião, surgiu a necessidade de acompanhar de forma mais individual as dúvidas e dificuldades no processo de mudança de estilo de vida. Dessa forma, foi feita uma divisão em que cada participante foi acompanhado por um acadêmico, por meio de conversas via remota. Ao final desse período, foi estruturado um relatório descrevendo como foi a experiência de cada dupla. 
Como resultados do projeto, constatou-se que dentre os 30 inscritos no projeto, 24 aderiram ao grupo do WhatsApp, dentre estes, 20 aderiram ao sistema de apadrinhamento. Entre os pacientes apadrinhados, 8 estavam com dificuldades para seguir uma dieta saudável, 7 estavam com dificuldades na realização de atividades físicas de modo regular e/ou quando as praticavam estas aconteciam sem o monitoramento de um educador físico e sem as precauções devidas, 5 estavam sem o acompanhamento periódico com um endocrinologista, 1 apresentava um quadro psicológico alterado desencadeado pelo medo das consequências da doença.

À medida que essas variáveis foram encontradas, os discentes buscaram estratégias para orientar melhor os participantes do projeto, para que eles pudessem contornar esses entraves que dificultavam o melhor controle da DM. Os pacientes com problemas relacionados à alimentação receberam dos alunos dicas de como poderiam aprimorar sua alimentação, monitorar a ingestão de carboidratos por meio do uso de aplicativos e a indicação de nutricionistas para ajudar na elaboração de dietas saudáveis. Já os indivíduos com dificuldades em praticar regularmente atividade física foram orientados a incorporar 30 minutos de exercício 5 vezes por semana, a procurar um educador físico para auxiliá-los.

Aos participantes que não realizavam um acompanhamento periódico com o endocrinologista, foi recomendado que procurassem um profissional para fazer o acompanhamento da doença e o contato de alguns médicos endocrinologistas foram disponibilizados. Para a paciente com quadro psicológico alterado, foi definida a estratégia de indicar o contato de um psicólogo que se colocou à disposição em acolher a participante do projeto.

Contudo, 5 participantes não retornaram as mensagens dos discentes via sistema de apadrinhamento, o que comprova que ainda persistem algumas dificuldades inerentes ao próprio paciente em e acolher o apoio fornecido pelos discentes envolvidos no projeto.

Ao final do projeto, foi aplicado um formulário anônimo via Google Forms para os pacientes, a fim de mensurar o impacto das atividades realizadas na vida deles e, também, para os organizadores do trabalho com a finalidade de avaliar a experiência de aprendizado desses alunos. Dentre os 16 participantes que responderam ao questionário, $68,75 \%$ relataram ter adotado hábitos de vida mais saudáveis, principalmente quanto à alimentação e à prática de exercícios, enquanto 31,25\% afirmaram que não tiveram alterações nos seus hábitos durante a realização do projeto, sendo justificados pelo contexto da pandemia e por falta de motivação. Ademais, dos 20 participantes que aderiram ao sistema de apadrinhamento, 14 responderam ao questionário, e dentre esses $92,85 \%$ avaliaram essa metodologia com a nota dez e 7,14\% com a nota um.

\section{DISCUSSÃO}

Diante da realidade de pandemia, a forma de contato entre profissionais da área da saúde e pacientes teve que ser adaptada e sofreu algumas alterações positivas para o enfrentamento desse novo momento (MARQUES LC, et al., 2020). Nesse contexto, a relação entre os estudantes e os participantes do projeto Diabetes em Foco também teve que passar por modificações e alterações metodológicas com o foco de dar viabilidade operacional ao projeto. Deste modo, ocorreram mudanças no preparo e no acolhimento dos portadores de DM e familiares que autorizaram sua inclusão no projeto.

Analisando os primeiros contatos com os integrantes, pôde-se observar que logo de início a adesão do grupo de diabéticos e suas famílias não foi integral, visto que dos 30 escolhidos uma parcela considerável não comparecia aos encontros. Nessa perspectiva, através do contato dos padrinhos (estudantes) e seus pacientes, foi possível concluir que parte destes não era familiarizada com os aplicativos utilizados, outra parcela abandonou o projeto sem deixar maiores informações sobre suas motivações. Entretanto, apesar do grupo que efetivamente comparecia às reuniões ter sido reduzido, de modo geral, o retorno dado por eles foi de contentamento e agradecimento pelas informações fornecidas.

No que se refere aos interesses que motivaram a busca dos pacientes pelo projeto, a maioria relatou 0 desejo de conhecer mais sobre a doença e os cuidados que precisam ser tomados após o diagnóstico de DM. Nesse sentido, é válido ressaltar que apesar de muitos grupos populacionais conviverem com a DM durante 
anos, poucos têm conhecimento básico sobre o assunto (BORBA AKOT, et al., 2015). Diante disso, cabe destacar que um baixo grau de escolaridade e uma bagagem de informações escassas sobre o assunto é fator de menor engajamento dos diabéticos no seu autocuidado e dificulta o manejo da doença (ALVARADOMAREL D, et al., 2019).

Em contrapartida, o uso de estratégias educativas que estimulem a construção de um conhecimento sobre diabetes pelos portadores da doença se mostra positivo, possibilitando melhorias no controle glicêmico (TORRES HC, et al., 2018). Desta maneira, torna-se imprescindível que o trabalho do profissional de saúde seja focado em fornecer informações claras ao paciente e sanar dúvidas, a fim de evitar possíveis complicações.

Em se tratando do autocuidado, os relatos dos participantes do projeto corroboram com estudos que indicam uma preferência por terapias medicamentosas, em contraste com a baixa adesão à dietas e ao exercício físico regular no tratamento da DM (GOMIDES DS, et al., 2013). Nesse cenário, destaca-se a importância de um acompanhamento profissional do doente, como ferramenta de incentivo a novas práticas de cuidado pessoal (BOELL JEW, et al., 2020). Além disso, uma série de estudos internacionais demonstrou a importância de abordagens educativas voltadas para mudanças de estilo de vida como forma efetiva de prevenção para o desenvolvimento de diabetes tipo 2 e de suas complicações (GRUSS SM, et al., 2019; LIAO LZ, et al., 2021).

Durante o acompanhamento, também se observou que o diagnóstico de diabetes afetou de forma negativa o estado emocional de alguns participantes. Assim, a ocorrência de casos de diabéticos que acordam durante a noite para checar diversas vezes a glicemia, por medo de uma crise hipoglicemia e outros que mudaram seu estilo de vida de forma drástica após o diagnóstico, demonstrou necessidade de um cuidado multiprofissional desses pacientes, ou seja, além de médicos, devem também ter apoio de nutricionistas, educadores físicos, enfermeiros, cirurgiões-dentistas, fisioterapeutas e psicólogos.

Desde a promulgação da Lei no 8080 , a saúde pública brasileira tem buscado evoluir para um cuidado integral ao paciente, ou seja, uma visão panorâmica do indivíduo e do processo saúde-doença, buscando distanciamento de uma abordagem estritamente fisiopatológica (BRASIL, 1990; SANTOS JUNIOR CJ, 2019). Além disso, diversos estudos nacionais têm apontado para a importância do desenvolvimento de habilidades de comunicação, empáticas e dialógicas de empoderamento no manejo de pacientes com doenças crônicas visando, em especial, o aumento da adesão ao tratamento (CYRINO AP, et al., 2009; FAVORETO CAO e CABRAL CC, 2009). Nesse contexto, uma formação médica que permita o desenvolvimento de tais habilidades é essencial.

Os alunos de medicina puderam desenvolver, no decorrer do projeto de extensão, habilidades comunicativas e empáticas ao manterem o contato com pacientes diabéticos, buscando compreender as complicações diretas e indiretas da doença no seu dia a dia, fornecendo informações sobre os mecanismos de instalação de complicações e, junto com o paciente, buscando maneiras de evitá-las através do aumento da adesão a um estilo de vida saudável e à prática de atividades físicas, bem como no direcionamento a profissionais de saúde qualificados.

\section{REFERÊNCIAS}

1. ALVARADO-MARTEL D, et al. Identification of psychological factors associated with adherence to self-care behaviors amongst patients with type 1 diabetes. Journal Diabetes Research, 2019; 14: 1-10.

2. BOELL JEW, et al. Resiliência e autocuidado em pessoas com diabetes mellitus. Texto e Contexto Enfermagem, 2020; 29: $1-12$

3. BOMFIM A. Impactos da pandemia na pesquisa científica: atuação da abc, coronavírus, webinários. 2020. Disponível em: http://www.abc.org.br/2020/07/21/impactos-da-pandemia-na-pesquisa-cientifica/. Acesso em: 17 jun. 2021.

4. BORBA AKOT, et al. Conhecimento sobre o diabetes e atitude para o autocuidado de idosos na atenção primária à saúde. Ciência \& Saúde Coletiva, 2019; 24(1): 125-136.

5. BRASIL. Lei no 8080 de 19 de setembro de 1990. Lei orgânica da saúde. 1990.

6. CORTEZ DN, et al.Complicações e o tempo de diagnóstico do diabetes mellitus na atenção primária. Acta Paulista de Enfermagem, 2015; 28(3): 255. 
7. CRUZ SC. Tratamento não farmacológico da Diabetes tipo 2. Rev. Port. Clin. Geral, 2005; 21: 587-595.

8. CYRINO AP, et al. Education for type 2 diabetes mellitus self-care: from compliance to empowerment. Interface (Botucatu), 2009; 13 (30): 93-106.

9. FARIA H, et al. Adesão ao tratamento em diabetes mellitus em unidades da Estratégia Saúde da Família. Revista da Escola de Enfermagem da USP, 2014; 48(2).

10. FAVORETO CAO, CABRAL CC. Narrativas sobre o processo saúde-doença: experiências em grupos operativos de educação em saúde. Interface (Botucatu), 2009; 13(28): 7-8.

11. FID. FEDERAÇÃO INTERNACIONAL DE DIABETES. Atlas de diabetes da Federação Internacional de Diabetes. 2019. Disponível em: <https://www.diabetesatlas.org>. Acesso em 21 de Dezembro de 2020.

12. GOMIDES DS, et al. Autocuidado das pessoas com diabetes mellitus que possuem complicações em membros inferiores. Acta Paulista de Enfermagem, 2013; 26(3): 289-293.

13. GRUSS SM, et al. Public Health Approaches to Type 2 Diabetes Prevention: the US National Diabetes Prevention Program and Beyond. Current Diabetes Reports, 2019; 19(9): 78.

14. LIAO LZ, et al. Causal effect of education on type 2 diabetes: A network Mendelian randomization study. World Journal of Diabetes, 2021; 12(3): 261-277.

15. MARQUES LC, et al. Covid-19: cuidados de enfermagem para segurança no atendimento de serviço pré-hospitalar móvel. Texto \& Contexto - Enfermagem. 2020; 29: 1-12.

16. OTERO LM,et al. Conhecimento do paciente diabético acerca de sua doença, antes e depois da implementação de um programa de educação em diabetes. Revista Latino-Americana de Enfermagem, 2008; 16(2): 231-237.

17. SANTOS ECB, et al. Os cuidados sob a ótica do paciente diabético e de seu principal cuidador. Revista LatinoAmericana de Enfermagem, 2005; 13(3): 397-406.

18. SANTOS JUNIOR CJ. Educação Médica e Formação na Perspectiva Ampliada e Multidimensional: Considerações acerca de uma Experiência de Ensino-Aprendizagem. Revista Brasileira de Educação Médica, 2019; 43(1): 72-79.

19. SBD. SOCIEDADE BRASILEIRA DE DIABETES. O que é diabetes? Disponível em: <https://www.diabetes.org.br/publico/diabetes/oque-e-diabetes>. Acesso em: 21 dez. 2020.

20. TORRES HC, et al. Evaluation of the effects of a diabetes educational program: a randomized clinical trial. Revista de Saúde Pública, 2018; 52.

21. ZAGURY L, et al. Diabetes sem medo. Rio de Janeiro: Rocco 4 ed, 113p. 